\title{
Respuesta a la carta al editor Tratamiento y desenlace en pancreatitis aguda
}

\section{Response to the letter to the editor Treatment and outcome in acute pancreatitis}

Leidy Juliana Puerto H. ${ }^{1 *}$, Luis Ramiro Núñez R. ${ }^{1}$

1 Hospital Universitario Hernando Moncaleano Perdomo de Neiva. Neiva, Colombia.

*Correspondencia: Leidy Juliana Puerto $\mathrm{H}$, puertojuliana@hotmail.com

Fecha recibido: $\quad 15 / 11 / 19$

Fecha aceptado: 19/11/19
Señor Editor:

Luego de leer con atención la carta de Abel Salvador Arroyo-Sánchez, MD, en respuesta al artículo publicado en su revista sobre 3 años manejo y desenlaces de la pancreatitis aguda, nos gustaría hacer algunos comentarios (1).

El primero es agradecer al médico Arroyo por su interés en aportar al conocimiento del tratamiento de esta enfermedad. Coincidimos con él en su apreciación acerca de los esquemas nutricionales recomendados en pancreatitis aguda por la mayoría de las guías basadas en la evidencia (2-4). Dichas guías claramente establecen los beneficios de la nutrición enteral en pacientes con pancreatitis aguda, dado esto, como se aprecia en la Tabla 12, más del $80 \%$ de los pacientes de nuestro trabajo recibió nutrición enteral o mixta; específicamente, en el caso de los pacientes con necrosis, el $62 \%$ recibió este tipo de nutrición (1). Es de anotar que en los pacientes con nutrición parenteral se agotaron las terapéuticas posibles para la vía enteral.

Como se aprecia en la Figura 2, nuestro trabajo exhibe un porcentaje elevado del $62,5 \%$ de cirugías realizadas a pacientes con necrosis pancreáticas. Dichas cirugías correspondían a complicaciones refractarias al tratamiento médico conservador y de pacientes que no fueron candidatos al manejo percutáneo ni endoscópico, por lo que se presenta desenlace mortal en 3 de los casos, quienes, como se aprecia en la Tabla 13, tenían una escala de severidad de Marshall $>4$ al ingreso y un promedio de APACHE II de 16 puntos, lo cual ensombrecía notablemente el pronóstico $(1,5,6)$.

Finalmente, consideramos que todos los aportes realizados a través de las cartas al editor demuestran el interés por el artículo y enriquecen el trabajo de investigación.

\section{REFERENCIAS}

1. Puerto LJ, Medina R, Núñez LR, Jiménez HC, Olaya JG, Sanjuán JF, et al. Outcomes of three years of experience managing acute pancreatitis at a fourth level hospital in Huila, Colombia. Rev Colomb Gastroenterol. 2019;34(1):10-6.

2. Crockett SD, Wani S, Gardner TB, Falck-Ytter Y, Barkun AN, Crockett S, et al. American Gastroenterological Association Institute Guideline on Initial Management of Acute Pancreatitis. Gastroenterology. 2018;154(4):1096-101. doi: 10.1053/j.gastro.2018.01.032.

3. Lodewijkx PJ, Besselink MG, Witteman BJ, Schepers NJ, Gooszen HG, van Santvoort HC, et al. Nutrition in acute pancreatitis: a critical review. Expert Rev 
Gastroenterol Hepatol. 2016;10(5):571-80. doi: 10.1586/17474124.2016.1141048.

4. McClave SA, Taylor BE, Martindale RG, Warren MM, Johnson DR, Braunschweig C, et al. Guidelines for the provision and assessment of nutrition support therapy in the adult critically ill patient: Society of Critical Care Medicine (SCCM) and American Society for Parenteral and Enteral Nutrition (A.S.P.E.N.). J Parenter Enter Nutr. 2016;40(2):159-211. doi: 10.1177/0148607115621863.
5. Yokoe M, Takada T, Mayumi T, Yoshida M, Isaji S, Wada $\mathrm{K}$, et al. Japanese guidelines for the management of acute pancreatitis: Japanese Guidelines 2015. J Hepatobiliary Pancreat Sci. 2015;22(6):405-32. doi: 10.1002/jhbp.259.

6. Leppäniemi A, Tolonen M, Tarasconi A, Segovia-Lohse H, Gamberini E, Kirkpatrick AW, et al. 2019 WSES guidelines for the management of severe acute pancreatitis. World J Emerg Surg. 2019;14:27. doi: 10.1186/s13017-019-0247-0. 